\title{
Non linéarité de l'essaim de Quito de 1998-1999 associé à l'activité du volcan Guagua Pichincha, Équateur
}

No linearidad del enjambre de Quito de 1998-1999 asociado a la actividad del volcán Guagua Pichincha, Ecuador

Non-linearity of the 1998-1999 Quito swarm associated to Guagua Pichincha volcano activity, Ecuador

Denis Legrand, Darwin Villagómez, Hugo Yepes et Alcinoe Calahorrano

\section{OpenEdition}

\section{Journals}

Édition électronique

URL : http://journals.openedition.org/bifea/6307

DOI : 10.4000/bifea.6307

ISSN : 2076-5827

Éditeur

Institut Français d'Études Andines

Édition imprimée

Date de publication : 1 avril 2003

Pagination : 1-22

ISSN : 0303-7495

Référence électronique

Denis Legrand, Darwin Villagómez, Hugo Yepes et Alcinoe Calahorrano, « Non linéarité de l'essaim de Quito de 1998-1999 associé à l'activité du volcan Guagua Pichincha, Équateur », Bulletin de l'Institut français d'études andines [En ligne], 32 (1) | 2003, mis en ligne le 08 avril 2003, consulté le 08 décembre 2020. URL : http://journals.openedition.org/bifea/6307 ; DOI : https://doi.org/10.4000/bifea.6307

\section{cc)}

Les contenus du Bulletin de l'Institut français d'études andines sont mis à disposition selon les termes de la licence Creative Commons Attribution - Pas d'Utilisation Commerciale - Pas de Modification 4.0 International. 


\title{
NON LINÉARITÉ DE L'ESSAIM DE QUITO DE 1998- 1999 ASSOCIÉ À L'ACTIVITÉ DU VOLCAN GUAGUA PICHINCHA, ÉQUATEUR
}

\author{
Denis LEGRAND*, Darwin VILLAGÓMEZ**, Hugo YEPES ${ }^{* *}$, \\ Alcinoe CALAHORRANO**
}

\begin{abstract}
Résumé
Les variations temporelles des dimensions multi-fractales spatiales de l'essaim de séismes tectoniques de Quito (Équateur) sont corrélées à l'activité du volcan Guagua Pichincha. Les dimensions multi-fractales diminuent avant les 5 pics d'énergie. Ces valeurs réaugmentent avant ou juste après le pic d'énergie. L'essaim de Quito est également caractérisé par de fortes valeurs du facteur $\mathrm{b}$ (variant de $1,12+/-0,05$ à $1,43+/-0,28$ ). Une rupture dans la courbe de la loi de Gutenberg-Richter est observée qui implique la présence de deux valeurs du facteur $b$. Nous supposons qu'il puisse s'agir d'un comportement catastrophique, où une bifurcation se produit et conduit à deux scenari volcaniques différents : un épisode éruptif ou un épisode de calme du Guagua Pichincha. Ce comportement peut dépendre de petites variations de conditions internes, telles que des variations du champ de contraintes et/ou de pression de pores associées à des mouvements de fluides (gaz, eau souterraine et/ou magma).
\end{abstract}

Mots clés :Dimensions multi-fractales, physique non linéaire du chaos, volcan Guagua Pichincha, essaim de Quito.

\section{NO LINEARIDAD DEL ENJAMBRE DE QUITO DE 1998-1999 ASOCIADO A LA ACTIVIDAD DEL VOLCÁN GUAGUA PICHINCHA, ECUADOR}

\section{Resumen}

Las variaciones temporales de las dimensiones espaciales multi-fractales del enjambre de sismos tectónicos de Quito (Ecuador) son correlacionadas con la actividad del volcán Guagua Pichincha. Las dimensiones multi-fractales disminuyen antes de los 5 picos de energía sísmica. Estos valores incrementan antes o justo después del pico de energía. El enjambre de Quito está también caracterizado por altos valores del factor $b$ (variando de $1,12+/-0,05$ hasta $1,43+/-0,28$ ). Una ruptura en la curva de la ley de Gutenberg-Richter occurió que implica la presencia de dos valores del factor $b$. Suponemos que puede ser un comportamiento catastrófico donde una

"IFEA : Institut Français d'Études Andines, Whymper 442 y Coruña, Quito ÉquateurE-mail : legrandenis@yahoo.com Ecuador.

*** IG-EPN : Instituto Geofísico de la Escuela Politécnica Nacional, apartado 2759, Quito, 
bifurcación puede conducir a 2 escenarios diferentes : un episodio eruptivo o un episodio de calma en el volcán Guagua Pichincha. Este comportamiento puede depender de pequeños cambios de condiciones internas, tal como variaciones del tensor de esfuerzos y/o de presión de poro asociadas a movimientos de fluidos (gas, agua subterránea y/o magma).

Palabras claves: Dimensiones multi-fractales, física no lineal del caos, volcán Guagua Pichincha, enjambre de Quito.

\title{
NON-LINEARITY OF THE 1998-1999 QUITO SWARM ASSOCIATED TO GUAGUA PICHINCHA VOLCANO ACTIVITY, ECUADOR
}

\begin{abstract}
Temporal variations of multi-fractal spatial dimensions of tectonic earthquakes of Quito swarm (Ecuador) are correlated to Guagua Pichincha volcano activity. Multi-fractal dimensions decrease before the 5 seismic energy peaks. These values increase before or just after the energy peak. Quito swarm is also characterized by high b-values (from 1,12+/- 0,05 to $1,43+/-0,28$ ). A break in the curve of the Gutenberg-Richter law occurred which implies the presence of two b-values. We suppose it may be a catastrophic behavior where a bifurcation may conduce to two different volcanic scenari : one eruptive period or one period of quiescence of Guagua Pichincha. This behavior may depend on small internal condition changes such as stress field and/or pore pressure variations associated to movement of fluids (gas, groundwater and/or magma).
\end{abstract}

Key words: Multi-fractal dimensions, non linear physics of chaos, Guagua Pichincha volcano, Quito swarm.

\section{INTRODUCTION}

Un essaim de séismes ayant les mêmes caractéristiques que des séismes tectoniques, à savoir ayant des ondes $\mathrm{P}$ et $\mathrm{S}$ claires avec un spectre dont les fréquences varient de 1 à $10 \mathrm{~Hz}$, a commencé avant l'activité phréatique du volcan GuaguaPichincha, Équateur, en 1998-1999 et avant son activité magmatique (Calahorrano et al., 1999 ; Calahorrano, 2001). Cet essaim est localisé au Nord de la ville de Quito, à environ 15-20 km au NE du volcan (Fig. 1). Il est apparu en juillet 1998, un mois avant une intense période d'activité phréatique du volcan, avec un second pic d'activité plus modéré en juin-août 1999. Le volcan Guagua Pichincha a eu par la suite une activité magmatique qui a commencé en septembre 1999 avec la formation d'une série de dômes dacitiques et des séismes volcaniques situés juste sous le volcan (Villagómez et al., 1999 ; Villagómez, 2000). Il faut noter l'occurence d'un séisme de magnitude $\underset{w}{\mathrm{w}}=7,1$ le 4 août 1998, à 18H59 GMT à Bahía de Caráquez, situé à 200 km à l'ouest du volcan, entre le commencement de l'essaim de Quito et l'intense période d'activité phréatique du volcan (Villagómez, 2000 ; Segovia, 2001). Il est difficile de prouver qu'il existe un lien de cause à effet entre les deux phénomènes (gros séisme et éruption du volcan), mais comme ils se produisirent presqu'en même temps et sont relativement proches, on peut supposer qu'il ne s' agit pas d'une simple coïncidence. Le but de cet article est de décrire et peut-être de comprendre un peu mieux la complexité de la dynamique du volcan en étudiant les valeurs du facteur $\mathrm{b}$ et des variations temporelles des dimensions multifractales $\mathrm{D}_{\mathrm{q}}$ de l'essaim de Quito. 


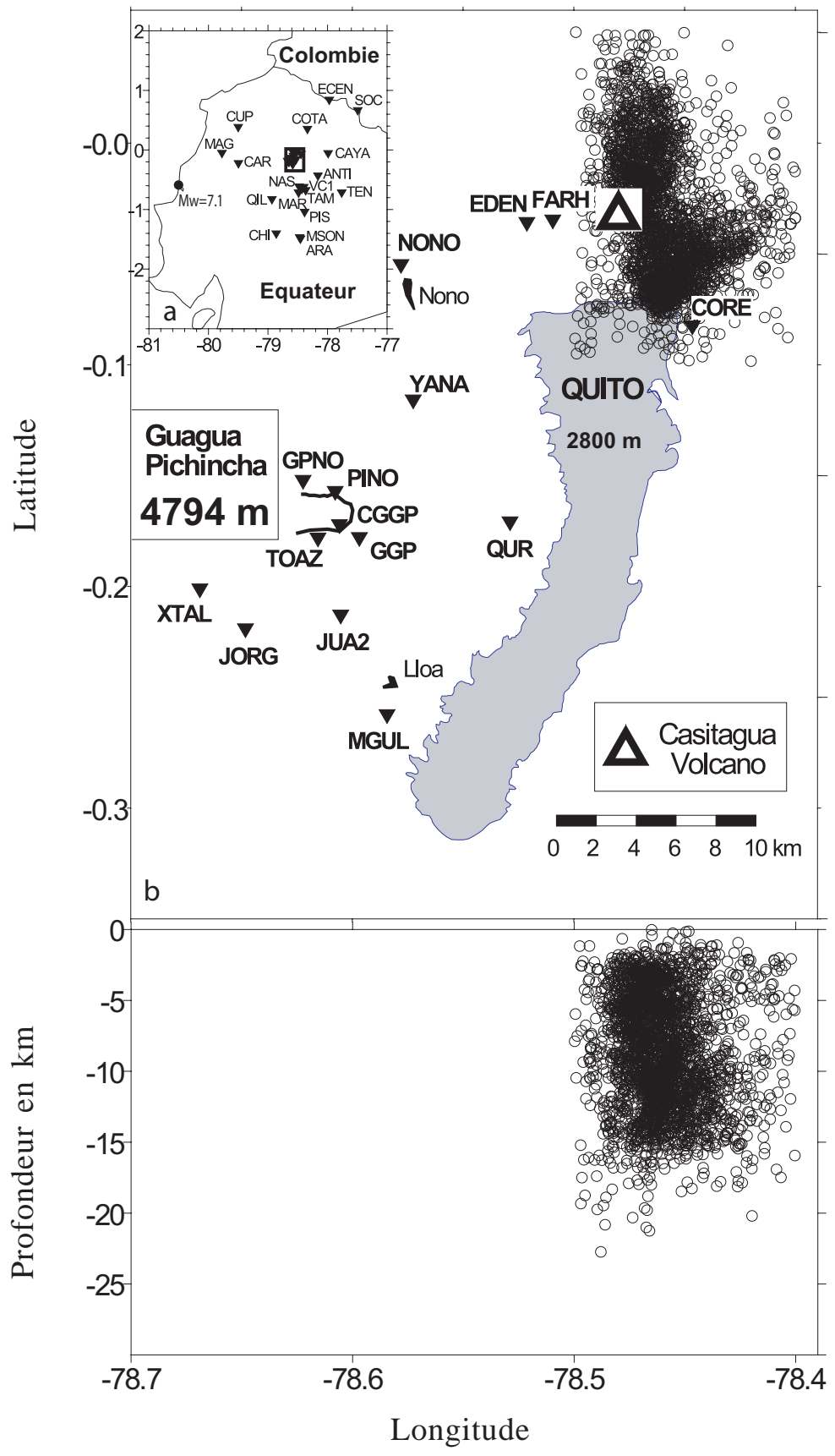

Fig. 1 - Stations sismiques régionales (Fig. 1a) et locales (Fig. 1b) (triangles) utilisées pour localiser les séismes de l'essaim de Quito avec la méthode de l'événement principal. 
Un essaim est constitué d'un nombre important de petits séismes par rapport aux gros séismes (i.e. sans un séisme caractéristique important), concentrés dans l'espace et le temps (Mogi, 1963). La valeur du facteur b associé à ce type d'activité sismique est souvent significativement plus grande que les valeurs moyennes correspondant aux séismes tectoniques qui varient entre 0,78 et 0,9 (Gutenberg \& Richter, 1949 ; 1954). De plus, les valeurs du facteur b sont souvent plus grandes dans les régions volcaniques que dans les régions tectoniques (Francis 1968 a ; b). Quelques exemples ont pour valeurs 1,3 au nord de la ride du milieu de l'Atlantique (Sykes, 1970), 1,24 pour l'effondrement de la caldera en 1968 dans les Îles volcaniques des Galapagos, Équateur (Francis, 1974), 1,2 +/- 0,1 pour l'essaim du volcan Mammoth Mountain en 1989, Californie (Hill et al., 1990), 1,2 pour l'essaim de la Péninsule d'Izu en 1989, Japon, associé à une éruption sous marine (Matsumura et al., 1991), et plus grand que 1.3 au volcan du Mt. St. Helens en 1988-1996, États-Unis (Wiemer \& McNutt, 1997). Néanmoins, des essaims avec de fortes valeurs du facteur b ne sont pas forcément spécifiques des seules régions volcaniques. Par exemple, une valeur de b de 1,3 +/- 0,1, avec $98 \%$ de confiance a été calculée pour l'essaim de Bucaramanga, Colombie (Rivera, 1989). De fortes valeurs de b $(1,2+/-0,11$ pour des profondeurs $<4 \mathrm{~km}$ et $1,28+/-0,12$ autour de $4 \mathrm{~km}$ de profondeur) ont été trouvées dans les parties superficielles du segment de Parkfield sur la faille de San Andreas (Wiemer \& Wyss, 1997).

Des variations spatiales et temporelles du facteur b ont été observées avant de gros séismes (e.g. Suyehiro et al., 1964 ;Mogi, 1969 ;Smith, 1981 ;Wyss \& Habermann, 1988) et autour de volcans (Wyss et al., 1997 ; Wiemer \& McNutt, 1997 ; Wiemert al., 1998 ; Poweret al., 1998). Séparer les aspects spatial et temporel dans de telles études est un véritable défi. Une tomographie tri-dimensionnelle (3D) du facteur b peut être réalisée au sein de petites cellules. Néanmoins, dans de tels cas, il n'est pas sûr que la loi de Gutenberg-Richter (à partir d'ici notée GR) soit respectée, i.e. que différentes tailles de séismes soient échantillonnées dans chacune des cellules (en particulier les gros événements ne seront pas présents dans de nombreuses cellules spatiales). Dans cet article, nous allons étudier uniquement les variations temporelles de distributions spatiales des séismes compris dans une grande région, sans résoudre le problème de fond d'interaction espace-temps (voir la discussion).

La complexité des distributions spatiales des séismes ne peut pas toujours être décrite par un paramètre unique comme le facteur $\mathrm{b}$ ou la dimension fractale $\mathrm{D}(e . g$. Kagan \& Knopoff, 1978 ; Ogata, 1988 ; Hirata, 1989). Il est parfois nécessaire de calculer un spectre de dimensions multi-fractales qui peut décrire un peu mieux cette complexité (e.g. Hirata \& Imoto, 1991 ; Dongshengt al., 1994). Nous allons montrer que l'essaim de Quito et les processus volcaniques ne sont pas de simples phénomènes linéaires, mais sont complexes et doivent être décrits par la physique non linéaire (physique du chaos).

\section{LE RÉSEAU SISMIQUE ET LA LOCALISATION DES SÉISMES}

Le réseau de stations sismologiques utilisé pour cette étude est composé de 35 stations courtes périodes parmi lesquelles 15 sont proches de l'essaim de Quito (Fig. 1). 
Les erreurs horizontales et verticales typiques sont de 850 et 2000 m respectivement. Un rapport de $\mathrm{Vp} / \mathrm{Vs}=1,68$ a été utilisé, sans variations claires avec la profondeur (Calahorrano, 2001).

Le calcul des dimensions multi-fractales spatiales requiert des localisations de séismes très précises car des erreurs de localisations peuvent introduire une surestimation de ces dimensions (Kagan \&Knopoff, 1980 ;Eneva, 1996). La technique de localisation, en utilisant un événement principal de référence dont la localisation est connue par ailleurs, (Spence, 1980 ; Besse, 1986) permet de calculer des localisations relatives avec une grande précision. Cette précision dépend des temps d'arrivées des ondes $\mathrm{P}$ et $\mathrm{S}$ et du modèle de vitesse. Comme les calculs des dimensions multi-fractales ne sont pas sensibles à des localisations absolues, une erreur sur la localisation absolue de l'événement principal ne va pas affecter les résultats.

Les séismes d'un essaim proviennent principalement d'une petite région, et par conséquent quelques unes des formes d'onde peuvent être très similaires. Ces événements, appelés multiplets, sont supposés avoir un même mécanisme au foyer. Poupinet et al. (1984) ont utilisé une telle similarité de la forme d'onde pour effectuer une relocalisation relative des événements par rapport à la localisation connue d'un événement. Ils calculèrent la phase entre les multiplets dans le domaine des fréquences, en utilisant une analyse inter-spectrale. Même si de nombreux événements de l'essaim de Quito peuvent être reconnus comme des multiplets, ce n'est pas le cas de tous, à cause surement de la différence de leurs mécanismes aux foyers (Legrand et al., 2002). Dans la mesure où nous essayons de traiter un catalogue complet, les relocalisations furent effectuées avec la technique de l'événement principal, et non pas à l'aide de l'approche des multiplets.

Les magnitudes locales furent calculées en utilisant la durée T (en sec) des sismogrammes à la station QUR, avec la formule classique (Lee et al., 1972) :

$$
\mathrm{M}_{\mathrm{L}}=-0,87+2,0 * \log _{10} \mathrm{~T}+0,0035^{*} \Delta
$$

où $\Delta$ est la distance hypocentrale (en $\mathrm{km}$ ). Presque tous les séismes ont été enregistrés à la station QUR. Si la lecture ne fut pas possible à cette station, elle a été réalisée à la station la plus proche du séisme enregistré.

\section{COMPILATION DU CATALOGUE}

Toute variation temporelle d'un paramètre nécessite un catalogue complet au cours du temps et une bonne connaissance d'acquisition des données. Toute corrélation entre des variations temporelles d'un paramètre avec tout changement de ses conditions d'acquisition peut être considérée comme suspecte. Par exemple, trouver une double pente de la loi de GR est toujours considéré comme suspect a priori, et une attention particulière doit être prise pour être sûr que cette brisure est réelle (Main, 2000). Des changements artificiels dans les taux d'activité sismique reliés à des changements de configuration du réseau sismique (installation de nouvelles stations ou fermeture de stations, changement des gains des instruments, changement des sismomètres, 
changement des seuils de magnitude, changement dans la façon de calculer la magnitude, etc.) peuvent affecter les calculs du paramètre b au cours du temps (Habermann, 1987 ; Eneva et al., 1994).

Deux pentes dans la loi de GR ont déjà été vues dans la caldera de Long Valley, Californie, pendant un essaim ayant une activité intense (Barton et al., 1999). Les auteurs expliquent cette cassure artificielle par l'impossibilité de compter tous les petits séismes pendant une activité sismique intense. Dans leur cas, le paramètre b correspondant à une activité intense des forts séismes est similaire au paramètre b d'un catalogue complet correspondant à une faible activité. En revanche, le paramètre b correspondant aux petits séismes est artificiellement petit, en raison d'une lecture incomplète due au fait que les séismes sont trop nombreux pour être tous détectés, et est plus petit que le paramètre $\mathrm{b}$ correspondant à un catalogue complet (pendant une activité sismique normale) pour le même intervalle de magnitudes.

\section{VALEURS DU PARAMÈTRE b DE L'ESSAIM DE QUITO}

Afin de considérer un catalogue complet, nous ne prenons en compte que la partie linéaire de la loi de GR. Dans le cas de l'essaim de Quito, cela correspond aux séismes ayant une magnitude locale supérieure à 1,4. Une double pente est observée dans la loi de GR de l'essaim de Quito. Nous allons essayer de prouver dans le prochain paragraphe que cette double pente n'est pas artificielle i.e. due à un catalogue incomplet ou à des interventions sur le réseau sismique.

La valeur b de la loi de GR est calculée par la méthode de vraisemblance maximum (Aki, 1965) :

$$
b=\frac{\log _{10} e}{\langle M\rangle-M_{\min }}
$$

où $M_{\text {min }}$ est la magnitude minimale à partir de laquelle la loi de GR est linéaire et $M$ est la moyenne des 'n' magnitudes correspondant à la partie linéaire de la loi de GR (magnitude plus grande que $M_{\text {mi }}$ ). Nous calculons l'erreur sur la valeur b avec une confiance de $95 \%$.

Sachant qu'il y a une interaction entre la taille de la région étudiée et la fenêtre temporelle d'étude (voir discussion), les fenêtres spatiale et temporelle ne peuvent pas être choisies de façon indépendante. Nous calculons les valeurs b pour tout le volume dans lequel sont contenus tous les séismes et la période complète mars 1998-décembre 1999.

Il est presque impossible de calculer des variations temporelles de b pour un essaim sur un petit intervalle de temps. Cela est dû au fait que les plus gros événements d'un essaim ne se situent pas au debut de l'activité mais sont répartis de façon aléatoire au cours du temps. Le calcul du facteur b nécessite une distribution des petits et gros séismes de telle sorte que la loi de GR soit satisfaite (i.e. présente une partie linéaire sur un intervalle fini de magnitudes). Cela suppose de prendre un long intervalle de temps afin d'échantillonner de façon correcte les petits et les gros séismes d'un essaim. À la 
suite de quoi, dans cet article, nous ne calculerons des valeurs de b que sur l'intervalle complet de temps.

\section{1. Double pente de la loi de GR de l'essaim de Quito : réelle ou non ?}

Deux nouvelles stations ont été installées au début de l'activité de l'essaim (FARH et EDEN), proche de la station CORE déjà existante (Fig. 1). Néanmoins, ces installations n'ont pas affecté le nombre de séismes de magnitude > 1,4 (le seuil de magnitude) qui a pu être enregistré par les autres 13 stations proches (incluant la station CORE). Ces deux nouvelles stations permettent d'avoir une meilleur précision dans la localisation des séismes, mais pas dans le comptage des séismes de magnitude supérieure à 1,4 .

Afin d'être sûr de la réalité de la double pente de la loi de GR, différentes étapes ont été suivies :

- La loi de GR est calculée sur l'ensemble de la région, incluant l'essaim de Quito (Fig. 2a). Une double pente dans la loi de GR est observée : la première avec une valeur $\mathrm{b}$ de $0,81+/-0,03$ entre $\mathrm{M}_{\mathrm{L}}=1,4$ et 2,6 et la deuxième avec une valeur $\mathrm{b}$ de $1,23+/-0,10$ entre $M_{L}=3,2$ et 5,2 .

- Afin de confirmer que la double pente est due à l'essaim de Quito, des calculs de b ont été effectués pour l'essaim de Quito seul (Fig. 2b). Une double pente est observée, une première avec une valeur de $1,12+/-0,05$ entre les magnitudes $M_{L}$ de 1,4 et 2,7, et une seconde de $1,43+/-0,28$ entre $M_{L}=2,7$ et 4,2 .

-Finalement, la loi de GR est calculée pour la région 1 - région 2 (Fig. 2c). Nous observons seulement une pente avec une valeur b de $1,12+/-0,09$ entre $M_{L}=3,1$ et 5,2. Cette valeur, identique à la pente de l'essaim de Quito pour les magnitudes de 1,4 à 2,7 (Fig. 2b, première pente), peut être considérée comme la valeur b régionale. Sur la figure $2 \mathrm{c}$ un second essaim est observé (celui de Pisayambo) qui ne montre pas de double pente. Par conséquent, le point 3 confirme que la brisure de la loi de GR n'existe pas en dehors de l'essaim de Quito, de telle sorte que cette brisure est bien associée à l'essaim de Quito.

La valeur b a été interprétée en terme de distribution géometrique de séismes comme une dimension fractale D (Aki, 1981) et en terme de tectonique (King, 1983). De nombreux cas de dimensions fractales ont été étudiés pour caractériser différentes sortes de régions sismiques et volcaniques (e.g. Kagan \& Knopoff, 1987 ; Turcotte, 1992). suivantes:

Aki (1981) a relié la valeur b à la dimension fractale $\mathrm{D}$ en utilisant les hypothèses

1-Le nombre d'événements suit la loi d'Ishimoto-Iida/Gutenberg-Richter (Ishimoto \& Iida, 1939 ; Gutenberg \& Richter, 1944):

$$
\log _{10} N(t)=a(t)-b M
$$

où $\mathrm{N}(\mathrm{t})$ est le nombre de séismes de magnitude $\geq M$ enregistrés pendant la période de temps $\mathrm{t}, \mathrm{a}(\mathrm{t})$ est le $\log _{10}$ du nombre de séismes de magnitude $\geq 0$ enregistrés pendant la 

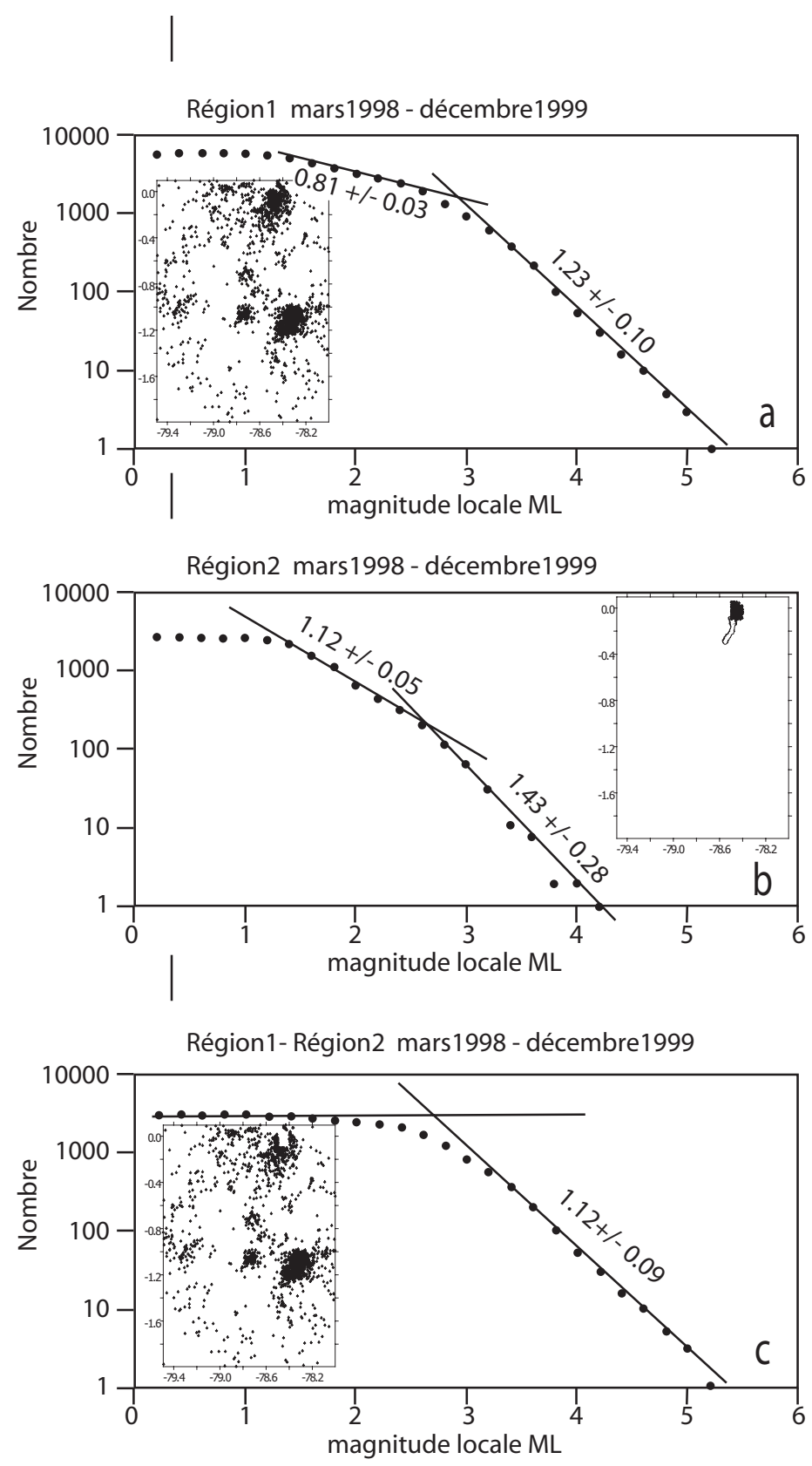

Fig. 2 - Loi de Gutenberg-Richter et sismicité correspondante pour :

a : la région 1 : incluant l'essaim de Quito et ses environs

b : la région 2 : seulement l'essaim de Quito

c : la région 1 - la région 2 
période de temps $t, \mathrm{~b}$ est une constante sur l'intervalle de temps t. Si nous considérons la fréquence $\log _{10} \mathrm{~N}(\mathrm{t}) / \mathrm{t}$, a $(\mathrm{t})$ devient dans la nouvelle loi fréquence-magnitude, le $\log _{10}$ du nombre de séismes de magnitude $\mathrm{M}=0$ par unité de temps et peut être considéré comme une constante au cours du temps.

2-Le moment sismique $\mathrm{M}_{0}$ est relié à la magnitude $\mathrm{M}$ par :

$$
\log _{10} M_{0}=c M+d
$$

où $\mathrm{c}$ et $\mathrm{d}$ sont des constantes.

3-La loi de similitude $\omega^{2}$ (Aki, 1967) est respectée pour les petits séismes. Cela suppose une chute de contrainte constante, et par conséquent le moment sismique $M_{0}=\mu S \Delta u \propto L^{3}$ où $\mu$ est le module de cisaillement, $S=$ LW est la surface de la faille avec $\mathrm{L}$ la longueur et $\mathrm{W}$ la largeur de la faille et $\Delta \mathrm{u}$ est la dislocation sur le plan de faille. Pour les petits séismes, cette propriété caractérise une auto-similarité dans les deux directions $\mathrm{L}$ et $\mathrm{W}$ (faille carrée avec $\mathrm{L} \approx \mathrm{W}$ ). Cette hypothèse est fausse pour les gros séismes pour lesquels la dimension W est bloquée, de telle sorte que, $M_{0} \propto L^{2}$ (Scholz, 1982 ; 1994).

Combinant 1,2 et 3 conduit à :

$$
N \propto L^{-\frac{3 b}{c}}
$$

La dimension fractale D est définie par Mandelbrot (1975) par $: N \propto L^{-D}$ donc Aki (1981) déduisit que $\mathrm{D}=3 \mathrm{~b} / \mathrm{c}=2 \mathrm{~b}$, pour une valeur typique de $\mathrm{c}=1,5$.

Dans le cas des essaims de séismes (i.e. pour les petits événements), le point 3 devrait être satisfait, mais il n'est pas sûr que $\mathrm{c}=1,5$. Donc la relation $\mathrm{D}=2 \mathrm{~b}$ ne peut pas être appliquée directement. Pour cette raison, dans cet article, b et D sont calculés indépendamment.

\section{VALEURS DE Dq POUR L'ESSAIM DE QUITO}

Les valeurs $b$ et $D_{q}$ ne peuvent pas être calculées sur les mêmes fenêtres temporelles parce que, comme déjà indiqué, dans le cas d'un essaim, les valeurs b doivent être calculées sur de grands intervalles de magnitudes (i.e. sur de longues fenêtres temporelles). En revanche, pour les calculs de $\mathrm{D}_{q}$, la magnitude n'entre pas en ligne de compte ; des fenêtres temporelles plus courtes peuvent donc être considérées. Les évolutions temporelles de $D_{q}$ sont calculées sur des fenêtres glissantes de 200 séismes, avec un recouvrement de 20 séismes. Il est à remarquer que dans ce mode de calcul, les fenêtres temporelles n'ont pas une durée constante. La table 1 donne les intervalles de temps pour chaque fenêtre. Cette démarche suppose qu'un souséchantillonnage d' un espace fractal est fractal, avec la même dimension fractale. Le fait d'utiliser un petit jeu de données (200) ne semble pas être un problème crucial si quelques précautions sont prises. Utiliser le jeu complet de données n'implique pas forcément une meilleure solution (Eneva, 1996). Ici, l'étude fut limitée à un jeu de 
données complet en magnitude correspondant aux séismes de magnitude locale supérieure à 1,4. Un problème plus critique est de travailler avec un jeu de données fini. En effet, un jeu de données infini devrait être utilisé pour être sûr du comportement multi-fractal. Dans quelques cas, un jeu mono-fractal peut apparaître comme multi-fractal seulement par le fait d'utiliser un jeu limité de données (Havstad \& Ehlers, 1989 ; Eneva, 1996). En fait, il est beaucoup plus facile de montrer un comportement multi-fractal (qui peut être artificiel dans certains cas) qu'un comportement mono-fractal, qui suppose d'utiliser un nombre infini de points, ce qui est impossible pour des données réelles (Eneva, 1996). Donc, dans cette étude, nous n'insisterons pas trop sur le caractère multi-fractal mais plus sur les variations temporelles de b et Dq qui semblent être un outil puissant pour détecter une forte activité sismique comme les expériences de cassures de roches en laboratoire (Hirata et al., 1987), pour les gros séismes (Radulian \& Trifu, 1991), pour les séismes qui se produisent dans les mines (Coughlin \& Kranz, 1991 ; Eneva, 1996) pour des catalogues synthétiques (Eneva \& Ben-Zion, 1994) ou pour des séquences de répliques (Legrand et al., 1996).

De nombreuses dimensions fractales ont été définies et nous en décrivons certaines ci-dessous (Eckmann \& Ruelle, 1985 ; Baker \& Gollub, 1990).

\section{1. La dimension de capacité $D_{C}$}

$\mathrm{D}_{\mathrm{C}}$ est définie comme :

$$
D_{C}=\lim _{\varepsilon \rightarrow 0} \frac{\log _{10} N(\varepsilon)}{\log _{10}(1 / \varepsilon)}
$$

où $\mathrm{N}(\varepsilon)$ est le nombre de boîtes non vides de taille e nécessaire pour couvrir l'objet fractal. Comme les boîtes couvrent le fractal, cette méthode de calcul de $\mathrm{D}_{\mathrm{C}}$ est souvent appelée méthode du comptage de boîtes. Ainsi cette définition prend en compte seulement le fait que les boîtes sont occupées ou non, quelque soit le nombre d'événements qu'il y a dans chaque boîte. $\mathrm{N}(\varepsilon)$ n'est pas pondéré par le nombre d'événements.

\section{2. La dimension d'information $D_{I}$}

$\mathrm{D}_{\mathrm{I}}$ est définie comme :

$$
D_{I}=\lim _{\varepsilon \rightarrow 0} \frac{I(\varepsilon)}{\log _{10} \varepsilon}=\lim _{\varepsilon \rightarrow 0} \frac{\sum_{i=1}^{N} p_{i} \log _{10} p_{i}}{\log _{10} \varepsilon}
$$

où $\mathrm{p}_{\mathrm{i}}=\mathrm{p}_{\mathrm{i}}(\varepsilon)$ est la probabilité d'occupation de la ième boîte de dimension e, et $S(\varepsilon)=-I(\varepsilon)=-\sum_{i=1}^{N} p_{i} \log _{10} p_{i}$ est l'entropie (moins l'information $\mathrm{I}(\varepsilon)$ ) et $\mathrm{N}$ le nombre de boîtes. La dimension d'information prend en compte le nombre de points dans chaque boîte que la dimension de capacité ne prenait pas en compte. Mais cette 
définition ne tient pas compte de la distribution de ces points dans chaque boîte (par exemple s'ils sont proches ou non les uns des autres).

Toutes ces définitions ont un rapport avec des boîtes. La position de la boîte initiale à partir de laquelle les calculs sont effectués est importante et le fait de changer la position de cette boîte initiale va affecter le résultat final. Donc, de nombreuses positions de la boîte initiale doivent être testées afin d'estimer l'influence de la boîte de départ sur le résultat final.

4. 3. La dimension de corrélation $D_{G}$

$\mathrm{D}_{\mathrm{G}}$ est définie comme :

$$
D_{G}=\lim _{\varepsilon \rightarrow 0} \frac{\log _{10} C(\varepsilon)}{\log _{10} \varepsilon}
$$

où

$$
C(\varepsilon)=\frac{1}{N} \sum_{j=1}^{N}\left[\frac{1}{N-1} \sum_{\substack{i=1 \\ i \neq j}}^{N} H\left(\varepsilon-\left\|\underline{x}_{i}-\underline{x}_{j}\right\|\right)\right]
$$

est la fonction de corrélation et $\mathrm{N}$ le nombre de points. $\left\|\underline{x}_{i}-\underline{x}_{j}\right\|$ est la distance entre les deux points $\underline{\underline{x}}_{i}$ et $\underline{x}_{j}$, et $\mathrm{H}$ est la fonction d'Heaviside. Cette définition fut introduite par Grassberger et Procaccia (1983) pour un signal temporel et fut ensuite généralisée à un jeu de points répartis dans l'espace. Cette définition prend en compte la distance entre chaque point, dont ne tenait pas compte les définitions précédentes. Cette définition ne faisant pas intervenir de boîte, le choix de la position de la boîte initiale ne se pose alors plus.

\section{4. Les dimensions fractales généralisées $D_{q}$}

$\mathrm{D}_{\mathrm{q}}$ sont définies comme (Grassberger, 1983 ; Hentschet \& Procaccia, 1983) :

$$
D_{q}=\frac{1}{q-1} \lim _{\varepsilon \rightarrow 0} \frac{\log _{10}\left(\sum_{i=1}^{N(\varepsilon)} p_{i}^{q}\right)}{\log _{10}(\varepsilon)}
$$

où $\mathrm{p}_{\mathrm{i}}=\mathrm{p}_{\mathrm{i}}(\mathrm{e})$ est la probabilité d'occupation de la ième boîte de dimension $\mathrm{e}$, et $\mathrm{q}$ est un nombre réel positif ou négatif. Cette définition prend en compte la distance généralisée d'ordre q entre les points, et peut être considérée comme la généralisation à l'ordre q de la dimension de corrélation. $p_{i}^{q}$ est la probabilité d'avoir q points dans la i ìme boîte, donc q peut être interprété comme le degré de corrélation. Enfin, $\mathrm{D}_{\mathrm{q}}$ peut être calculé de la même manière que la dimension de corrélation (Kurthz \& Herzel, 1987 ; Hirata \& Imoto, 1991 ; Eneva, 1994) :

$$
D_{q}=\lim _{\varepsilon \rightarrow 0} \frac{\log _{10} C_{q}(\varepsilon)}{\log _{10} \varepsilon}
$$


où:

$$
C_{q}(\varepsilon)=\left\{\frac{1}{N} \sum_{j=1}^{N}\left[\frac{1}{N-1} \sum_{\substack{i=1 \\ i \neq j}}^{N} H\left(\varepsilon-\left\|\underline{x}_{i}-\underline{x}_{j}\right\|\right)\right]^{(q-1)}\right\}^{\frac{1}{q-1}}
$$

Ainsi nous avons : $\mathrm{D}=\mathrm{D}_{\mathrm{C}}, \mathrm{D}_{1}=$ Det $\mathrm{D}_{2}=\mathrm{D}_{\text {. }}$.

$\mathrm{D}_{\mathrm{q}}$ est une fonction décroissante de $\mathrm{q}$, i.e. si $\mathrm{p}<\mathrm{q}$ alors $\mathrm{D}_{\mathrm{p}}>\mathrm{D}_{\mathrm{q}}$.

Pour un simple mono-fractal, toutes ces définitions sont identiques, à savoir $\mathrm{D}_{\mathrm{q}}$ est indépendant de q.

Comme nous l'avons montré, toutes ces définitions ne prennent pas en compte la magnitude, donc nous supposons une auto-similarité des événements par rapport à la magnitude. Un modèle simple de points sources est également utilisé pour chaque séisme, indépendamment de la magnitude. Il serait préférable de prendre en considération la taille de chaque faille ainsi que son orientation dans l'espace, mais cela suppose de connaître son mécanisme au foyer, ce qui est quasi impossible pour les petits séismes.

La figure 3 montre un exemple de la fonction de corrélation $\mathrm{C}_{\mathrm{q}}$ en fonction de $\mathrm{e}$ pour la période 1 , pour $\mathrm{q}=2$ à $\mathrm{q}=25$. La figure 4 montre toutes les variations temporelles des dimensions multi-fractales $\mathrm{D}_{\mathrm{q}}$ pour $\mathrm{q}=2$ à $\mathrm{q}=25$. La figure 5 montre un exemple de spectre multi-fractal pour la période 1 .

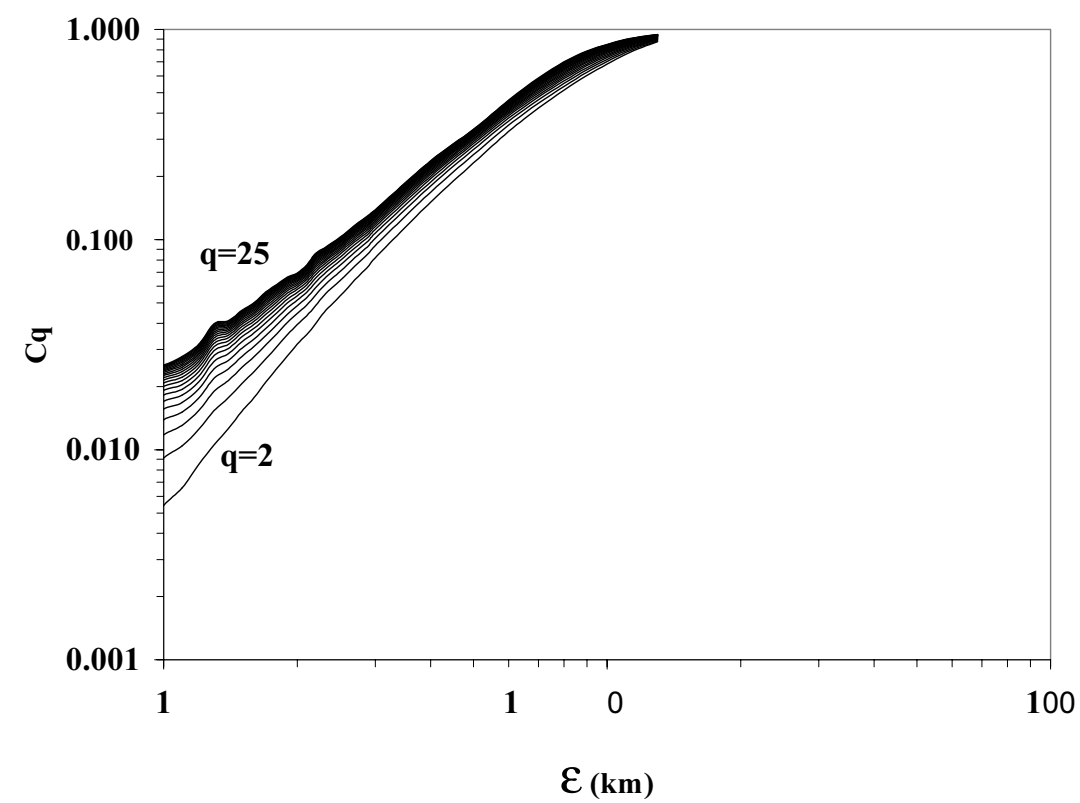

Fig. 3 - Exemple de fonction de corrélation en fonction d'epsilon pour la période 1 (voir Table 1). 


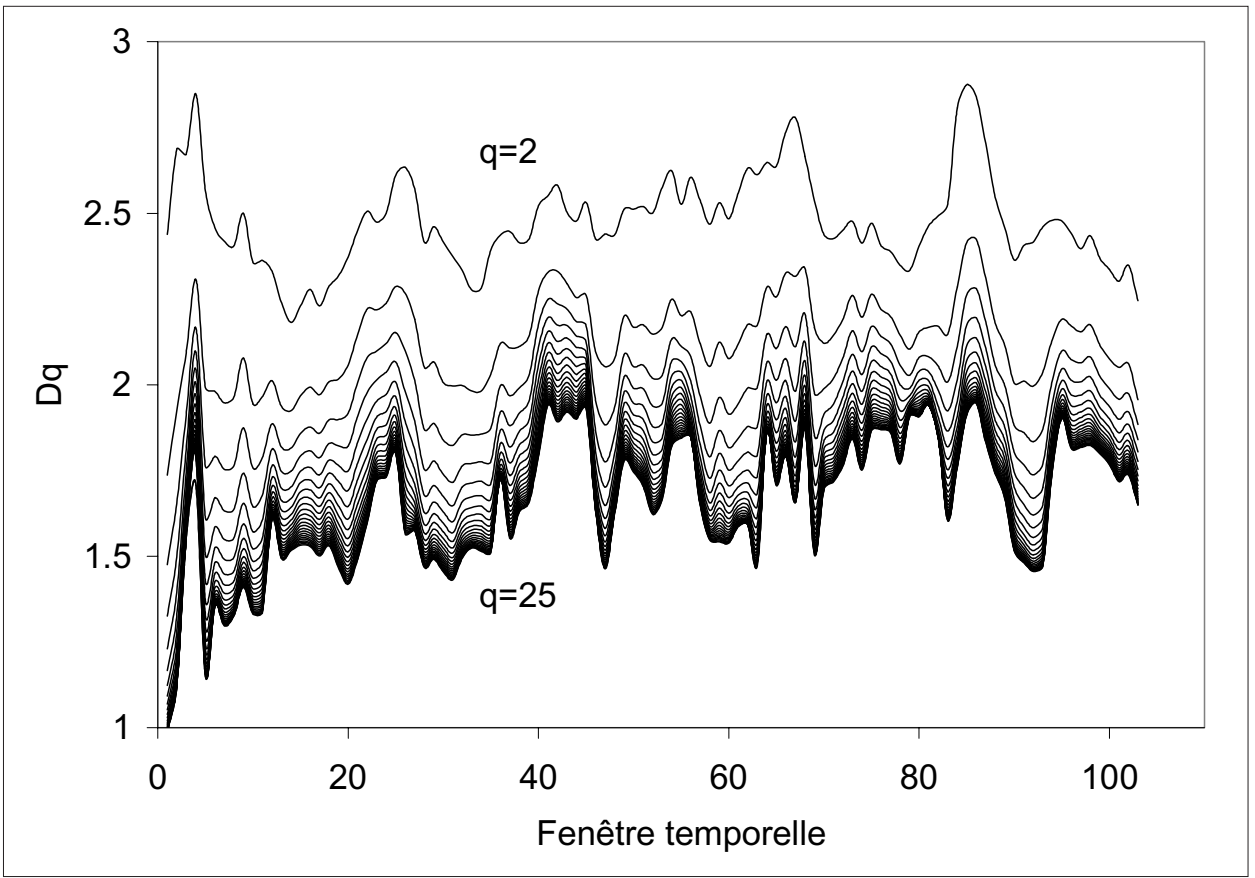

Fig. 4 - Dimensions multi-fractales Dq pour q=2 à 25 en fonction des fenêtres temporelles (voir Table 1).

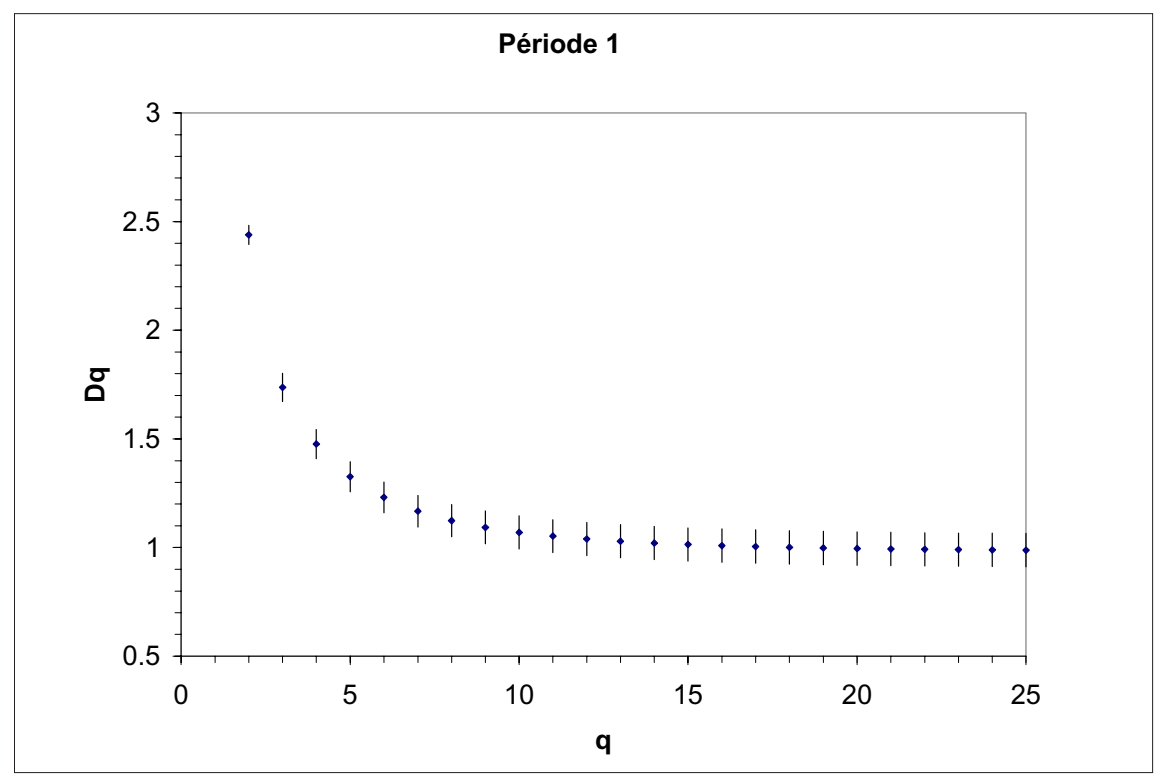

Fig. 5 - Exemple d'un spectre multi-fractal de Dq en fonction de q pour q=2 à 25, pour la période 1 (voir Table 1). 
Table 1 - Fenêtres temporelles (périodes) de l'essaim de Quito correspondant chacune à 200 événements de magnitude plus grande que 1,4.

\begin{tabular}{|c|c|}
\hline Period & Temporal window \\
\hline 1 & $98 / 03 / 08-98 / 07 / 25$ \\
\hline 2 & $98 / 03 / 29-98 / 07 / 26$ \\
\hline 3 & $98 / 04 / 15-98 / 07 / 27$ \\
\hline 4 & $98 / 06 / 23-98 / 07 / 30$ \\
\hline 5 & $98 / 06 / 26-98 / 07 / 30$ \\
\hline 6 & $98 / 07 / 09-98 / 07 / 31$ \\
\hline 7 & $98 / 07 / 12-98 / 07 / 31$ \\
\hline 8 & 98/07/13-98/08/01 \\
\hline 9 & $98 / 07 / 15-98 / 08 / 02$ \\
\hline 10 & $98 / 07 / 24-98 / 08 / 02$ \\
\hline 11 & $98 / 07 / 25-98 / 08 / 03$ \\
\hline 12 & $98 / 07 / 26-98 / 08 / 05$ \\
\hline 13 & $98 / 07 / 27-98 / 08 / 05$ \\
\hline 14 & $98 / 07 / 30-98 / 08 / 05$ \\
\hline 15 & $98 / 07 / 30-98 / 08 / 06$ \\
\hline 16 & $98 / 07 / 31-98 / 08 / 06$ \\
\hline 17 & $98 / 07 / 31-98 / 08 / 07$ \\
\hline 18 & 98/08/01-98/08/07 \\
\hline 19 & $98 / 08 / 02-98 / 08 / 08$ \\
\hline 20 & 98/08/02-98/08/09 \\
\hline 21 & $98 / 08 / 03-98 / 08 / 09$ \\
\hline 22 & $98 / 08 / 05-98 / 08 / 10$ \\
\hline 23 & $98 / 08 / 05-98 / 08 / 10$ \\
\hline 24 & $98 / 08 / 05-98 / 08 / 11$ \\
\hline 25 & $98 / 08 / 06-98 / 08 / 12$ \\
\hline 26 & $98 / 08 / 06-98 / 08 / 12$ \\
\hline 27 & $98 / 08 / 07-98 / 08 / 12$ \\
\hline 28 & $98 / 08 / 07-98 / 08 / 13$ \\
\hline 29 & $98 / 08 / 08-98 / 08 / 14$ \\
\hline 30 & $98 / 08 / 09-98 / 08 / 15$ \\
\hline 31 & $98 / 08 / 09-98 / 08 / 16$ \\
\hline 32 & $98 / 08 / 10-98 / 08 / 17$ \\
\hline 33 & $98 / 08 / 10-98 / 08 / 18$ \\
\hline 34 & $98 / 08 / 11-98 / 08 / 18$ \\
\hline 35 & $98 / 08 / 12-98 / 08 / 19$ \\
\hline 36 & $98 / 08 / 12-98 / 08 / 20$ \\
\hline 37 & $98 / 08 / 12-98 / 08 / 20$ \\
\hline 38 & $98 / 08 / 13-98 / 08 / 21$ \\
\hline 39 & $98 / 08 / 14-98 / 08 / 22$ \\
\hline 40 & $98 / 08 / 15-98 / 08 / 22$ \\
\hline 41 & $98 / 08 / 16-98 / 08 / 23$ \\
\hline 42 & $98 / 08 / 17-98 / 08 / 25$ \\
\hline 43 & $98 / 08 / 18-98 / 08 / 27$ \\
\hline 44 & $98 / 08 / 18-98 / 08 / 28$ \\
\hline 45 & $98 / 08 / 19-98 / 08 / 30$ \\
\hline 46 & $98 / 08 / 20-98 / 08 / 31$ \\
\hline 47 & $98 / 08 / 20-98 / 08 / 31$ \\
\hline 48 & $98 / 08 / 21-98 / 09 / 01$ \\
\hline 49 & $98 / 08 / 22-98 / 09 / 01$ \\
\hline 50 & $98 / 08 / 22-98 / 09 / 02$ \\
\hline 51 & $98 / 08 / 23-98 / 09 / 02$ \\
\hline 52 & $98 / 08 / 25-98 / 09 / 03$ \\
\hline
\end{tabular}

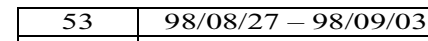

\begin{tabular}{|l|l|}
\hline 53 & $98 / 08 / 27-98 / 09 / 03$ \\
\hline 54 & $98 / 08 / 28-98 / 09 / 04$ \\
\hline
\end{tabular}

\begin{tabular}{|l|l|}
\hline 55 & $98 / 08 / 30-98 / 09 / 05$ \\
\hline 56 & $98 / 08 / 31-98 / 09 / 05$ \\
\hline
\end{tabular}

\begin{tabular}{|l|l|}
\hline 56 & $98 / 08 / 31-98 / 09 / 05$ \\
\hline 57 & $98 / 08 / 31-98 / 09 / 07$ \\
\hline
\end{tabular}

\begin{tabular}{|l|l|}
\hline 57 & $98 / 08 / 31-98 / 09 / 07$ \\
\hline 58 & $98 / 09 / 01-98 / 09 / 09$ \\
\hline
\end{tabular}

\begin{tabular}{|l|l|}
\hline 59 & $98 / 09 / 01-98 / 09 / 09$ \\
\hline 60 & $98 / 09 / 02-98 / 09 / 13$ \\
\hline
\end{tabular}

\begin{tabular}{|c|c|}
\hline 61 & $98 / 09 / 02-98 / 09 / 16$ \\
\hline 62 & $98 / 09 / 03-98 / 09 / 20$ \\
\hline
\end{tabular}

\begin{tabular}{l|l}
62 & $98 / 09 / 03-98 / 09 / 20$ \\
\hline
\end{tabular}

\begin{tabular}{l|l}
63 & $98 / 09 / 03-98 / 09 / 25$
\end{tabular}

\begin{tabular}{l|l}
64 & $98 / 09 / 04-98 / 09 / 30$ \\
\hline 65 & $98 / 09 / 05-98 / 10 / 04$
\end{tabular}

\begin{tabular}{l|l}
65 & $98 / 09 / 05-98 / 10 / 04$
\end{tabular}

\begin{tabular}{l|l}
66 & $98 / 09 / 05-98 / 10 / 09$ \\
\hline 67 & $98 / 09 / 07-98 / 10 / 12$
\end{tabular}

\begin{tabular}{l|l}
68 & $98 / 09 / 09-98 / 10 / 15$ \\
\hline 69
\end{tabular}

\begin{tabular}{l|l}
69 & $98 / 09 / 09-98 / 10 / 16$ \\
\hline
\end{tabular}

\begin{tabular}{l|l}
70 & $98 / 09 / 13-98 / 10 / 18$
\end{tabular}

\begin{tabular}{l|l}
71 & $98 / 09 / 16-98 / 10 / 20$ \\
\hline 72 & $98 / 09 / 20-98 / 10 / 21$
\end{tabular}

\begin{tabular}{|c|c|}
\hline 72 & $98 / 09 / 20-98 / 10 / 21$ \\
\hline 73 & $98 / 09 / 25-98 / 10 / 23$ \\
\hline
\end{tabular}

\begin{tabular}{l|l}
73 & $98 / 09 / 25-98 / 10 / 23$ \\
\hline 74 & $98 / 10 / 01-98 / 10 / 24$
\end{tabular}

\begin{tabular}{|c|c|}
\hline 75 & $98 / 10 / 04-98 / 10 / 26$ \\
\hline 76 & $98 / 10 / 09-98 / 10 / 28$ \\
\hline
\end{tabular}

\begin{tabular}{l|l}
76 & $98 / 10 / 09-98 / 10 / 28$ \\
\hline 77 & $98 / 10 / 12-98 / 11 / 04$
\end{tabular}

\begin{tabular}{|l|l|}
\hline 78 & $98 / 10 / 15-98 / 11 / 30$ \\
\hline 79 & $98 / 10 / 16-98 / 12 / 28$
\end{tabular}

\begin{tabular}{|c|c|}
\hline 80 & $98 / 10 / 18-99 / 02 / 06$ \\
\hline 81 & $98 / 10 / 20-99 / 03 / 04$ \\
\hline
\end{tabular}

\begin{tabular}{l|l}
81 & $98 / 10 / 20-99 / 03 / 04$ \\
\hline 82 & $98 / 10 / 21-99 / 03 / 24$
\end{tabular}

\begin{tabular}{c|c}
82 & $98 / 10 / 21-99 / 03 / 24$ \\
\hline 83 & $98 / 10 / 23-99 / 04 / 14$ \\
\hline
\end{tabular}

\begin{tabular}{l|l}
84 & $98 / 10 / 24-99 / 04 / 28$
\end{tabular}

\begin{tabular}{l|l}
85 & $98 / 10 / 26-99 / 05 / 13$ \\
\hline
\end{tabular}

\begin{tabular}{l|l}
86 & $98 / 10 / 28-99 / 06 / 16$ \\
\hline 87 & $98 / 11 / 04-99 / 06 / 27$
\end{tabular}

\begin{tabular}{l|l}
87 & $98 / 11 / 04-99 / 06 / 27$ \\
\hline 88 & $98 / 11 / 30-99 / 06 / 27$
\end{tabular}

\begin{tabular}{l|l}
89 & $98 / 12 / 28-99 / 07 / 08$
\end{tabular}

\begin{tabular}{l|l}
90 & $99 / 02 / 06-99 / 07 / 12$ \\
\hline
\end{tabular}

\begin{tabular}{l|l}
91 & $99 / 03 / 04-99 / 07 / 15$ \\
\hline 92 & $99 / 03 / 24-99 / 07 / 16$
\end{tabular}

\begin{tabular}{l|l}
93 & $99 / 04 / 20-99 / 07 / 18$
\end{tabular}

\begin{tabular}{l|l}
94 & $99 / 04 / 29-99 / 07 / 19$ \\
\hline 95 & $99 / 05 / 15-99 / 07 / 20$
\end{tabular}

\begin{tabular}{l|l}
96 & $99 / 06 / 16-99 / 07 / 21$
\end{tabular}

\begin{tabular}{l|l}
97 & $99 / 06 / 27-99 / 07 / 23$ \\
\hline
\end{tabular}

\begin{tabular}{l|l}
98 & $99 / 06 / 27-99 / 07 / 31$
\end{tabular}

\begin{tabular}{l|l}
99 & $99 / 07 / 09-99 / 08 / 26$
\end{tabular}

$100 \quad 99 / 07 / 12-99 / 08 / 30$

\begin{tabular}{l|l}
101 & $99 / 07 / 15-99 / 09 / 29$ \\
\hline 102 & $99 / 07 / 16-99 / 10 / 14$
\end{tabular}

\begin{tabular}{l|l}
102 & $99 / 07 / 16-99 / 10 / 14$ \\
103 & $99 / 07 / 18-99 / 11 / 16$
\end{tabular}

Les variations temporelles des dimensions multi-fractales $\mathrm{D}_{2}$ sont comparées à l'énergie sismique (Fig. 6). Avant chacun des 5 pics les plus énergétiques d'activité, les dimensions multi-fractales (nous ne montrons que $\mathrm{D}_{2}$ pour la clarté du graphique) décroissent. Elles croissent ensuite jusqu'à revenir à leur niveau d' origine avant ou juste après le maximum du pic. Les pics 4 et 5 sont proches, de telle sorte que $\mathrm{D}_{2}$ n'a pas le temps de recroître suffisamment et décroît avant le pic 5. La période de calme entre les pics 2 et 3 est décrit par des dimensions multi-fractales constantes. 


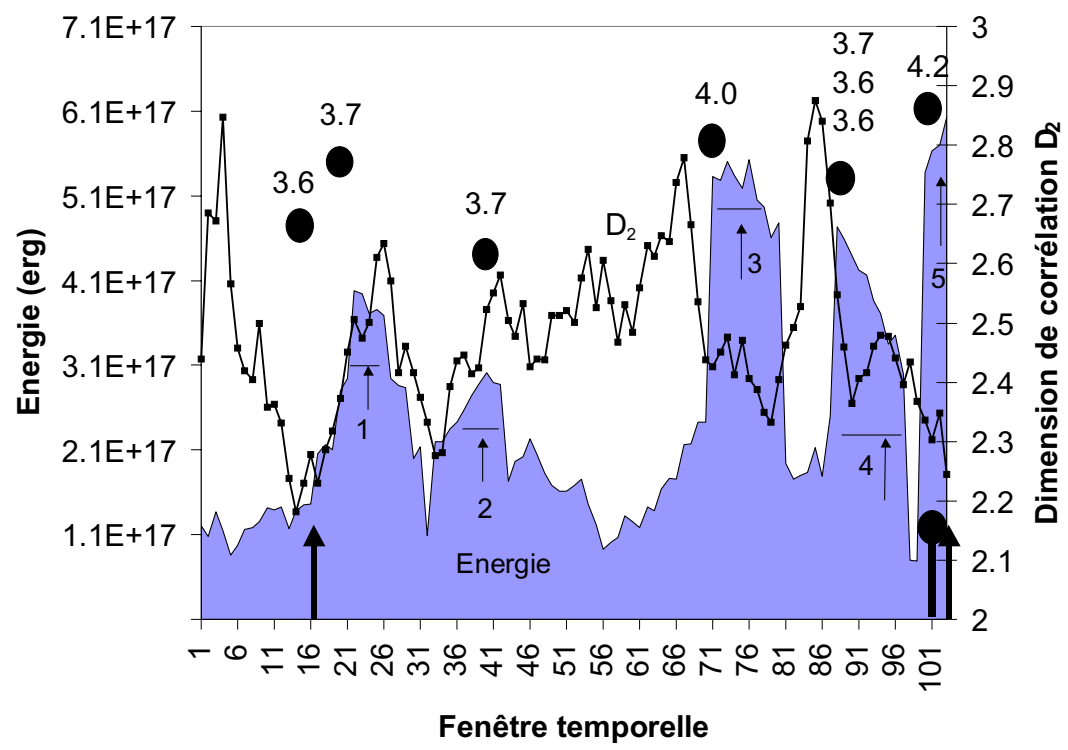

Fig. 6 - Variations temporelles de l'énergie (zone ombrée) et dimension multifractale $\mathrm{D}_{2}$ (ligne continue) avec les 6 principales magnitudes (points noirs). Les deux flèches verticales correspondent d'une part à la première éruption phréatique (période 17), et d'autre part aux principales explosions phréatiques du 5 et 7 octobte 1999 (période 102). La ligne verticale avec un point correspond à l'extrusion du premier dôme de lave entre le 25 et 30 septembre 1999 (périodes 99 et 100).

\section{DISCUSSION}

La loi de GR a largement été vérifiée pour des séquences de choc principal/ répliques, avec des valeurs classiques du paramètre b proche de 1. Quand un gros séisme se produit, le milieu élastique réagit à cette excitation sans force extérieure. Les répliques s'auto-organisent par rapport à la magnitude. Ceci peut être dû à la réponse du sol plus ou moins élastique suite au choc principal qui peut être décrit en première approximation par une force impulsive. Un tel système est appelé un système clos, parce qu'il n'y a pas d'énergie apportée au système.

Pour un volcan, la situation est différente. De l'énergie additionnelle [comme de l'énergie thermique générée par du magma ou de l'eau sous pression, du gaz, générant une contrainte thermo-élastique (Warren \& Latham, 1970)] peut être apportée au système, décrit comme un système ouvert. Cette énergie externe entretient le système, générant de nombreux petits séismes de taille comparable, sans provoquer un gros choc principal comme pour les séismes tectoniques. De nombreux petits événements peuvent être générés par des variations de contraintes sous le volcan dues à des mouvements de fluides par exemple, provoquant de grandes valeurs du paramètre b. Dans certains cas, des valeurs de b plus grandes que 1,5 (parfois pouvant atteindre 2,6) ont été évoquées 
(e.g. McNutt, 1986). Si nous relions la valeur b à la dimension fractale $\mathrm{D}$ suivant Aki (1981), $\mathrm{D}=2 \mathrm{~b}$, comme l'espace doit vérifier $\mathrm{D} \leq 3$, en théorie b devrait être plus petit que 1,5 . Une valeur de $\mathrm{b}$ supérieure à 1,5 signifie qu'il est impossible d'interpréter $\mathrm{b}$ comme une répartition spatiale de séismes via la dimension fractale. Ainsi b et D doivent être calculées et interprétées indépendamment (e.g. Barton et al., 1999). Pour un essaim volcanique, il est important d'utiliser la physique non-linéaire en plus des études linéaires classiques. Cette complexité d' auto-organisation spatio-temporelle des séismes est due à des processus physiques fondamentaux comme suggérés par Sornette et Sornette (1999) et Wesnousky (1999).

Ces systèmes fermés/ouverts peuvent être vus sur la forme des sismogrammes. Pour un séisme typique le sismogramme montre une onde $\mathrm{P}$ impulsive suivie par une décroissance de l'amplitude avec le temps. Cette observation suppose une réponse libre du sol à une impulsion courte du séisme. Dans le cas d'événements volcaniques (trémor, éruptions phréatique et/ou magmatique), le signal sismique est complètement différent. Le signal ne montre pas de décroissance régulière de l'amplitude avec le temps, mais l'amplitude peut rester constante comme pour le trémor volcanique et les éruptions phréatiques qui apparaissent souvent comme la superposition de nombreux petits événements élémentaires générant des signaux de longue durée. Cela signifie que le milieu ne répond pas à une seule force mais à une histoire entretenue de la source sismique. Comparant un événement unique et un essaim dans son ensemble, nous concluons que l'essaim n'est pas la réponse à une excitation unique (comme un choc principal suivi de nombreuses répliques) mais le résultat d'une source entretenue, comme le mouvement de fluides sous pression, des changements de contraintes...

Il existe une dualité entre le temps et l'espace dans les calculs du facteur b. Cela signifie que pour satisfaire la loi de GR, on peut considérer une petite région observée sur un laps de temps important, ou on peut considérer une région plus grande sur un intervalle de temps plus court. La taille de la région où b est calculé doit être choisie en respectant certains critères : par exemple, si la magnitude maximale enregistrée est $M_{\text {ma }}$, la taille de la région sur laquelle est calculé b doit être au moins égale à la taille caractéristique du séisme de magnitude $M_{\text {ma }}$, pour être sûr d'enregistrer toutes les répliques associées à ce séisme. Une fois la fenêtre spatiale choisie, la fenêtre temporelle doit être suffisamment longue pour échantillonner toutes les magnitudes dans cette fenêtre temporelle. Cette hypothèse est parfois difficile à respecter dans la pratique. Une alternative est de fixer un nombre d'événements assez grand pour obtenir un échantillonnage en magnitude suffisant. Ce n'est pas toujours le cas pour les essaims. Un problème crucial existe quand l'espace est découpé en petites cellules à l'intérieur desquelles b est calculé : l'utilisation d'un nombre limité de données peut provoquer des erreurs d'estimation de ce facteur b (Eneva, 1996). Il n'est pas sûr que chaque cellule ait le même nombre d'événements. Ainsi la loi de GR n'est pas forcément homogène dans l'espace, et dans certaines cellules elle ne sera pas respectée à cause du nombre limité de données. Il peut être plus utile de considérer des dimensions multi-fractales qui prennent en compte des distances entre séismes et non des magnitudes.

À l'aplomb d'un volcan, on considère généralement une petite région, ce qui implique théoriquement une longue fenêtre temporelle afin d'être sûr d'enregistrer toute 
l'activité (en particulier les gros événements) de cette région. Cette hypothèse est rarement vérifiée, parce que les gros événements se produisent rarement sous les volcans. Néanmoins, il y a des exceptions. Quelques uns ont été enregistrés proche de certains volcans : M=7,1 en 1975 proche du volcan Kalapana (États-Unis), M= 7,0 en 1912 proche du volcan Katmai (États-Unis), $M_{s}=7,0$ en 1914 sous le volcan Sakurajima (Japon), $M_{\mathrm{JMA}}=6,4$ en 1982 proche du volcan Miyake-jima (Japon), $\mathrm{M}_{\mathrm{s}}=6,3$ en 1916 proche du volcan Asama (Japon), $M_{1}=6,2$ en 1980 à Long Valley (États-Únis), $M_{\mathrm{s}}=6,2$ en 1983 sous le Miyake-jima (Japon), $M=6,0$ en 1975 proche du volcan Aso (Japon), $M_{s}=5,7$ en 1968 proche du volcan Fernandina (Équateur), $M_{s}=5,6$ en 1991 proche du volcan Sabancaya (Pérou) (McNutt, 2000). Donc, de grandes valeurs de b sous les volcans peuvent être, dans certains cas, dues à de petites fenêtres temporelles.

Des grandes valeurs de b supérieures à 1 reflètent une activité importante des petits séismes par rapport aux gros séismes. Différentes explications ont été proposées. Mogi (1962) a suggéré que des matériaux fortement hétérogènes soumis à un chargement mécanique peuvent générer de grandes valeurs de b. Scholz (1968) et Wyss (1973) proposèrent que de grandes valeurs de b étaient plus sûrement liées à une décroissance de l'amplitude des contraintes appliquées à l'échantillon de roche : “ les fractures auront toujours tendance à être plus petites, et b plus grand " (Scholz, 1968). La valeur b peut être corrélée à la nature des roches : une roche ductile a une valeur de $b$ plus grande qu' une roche cassante (Scholz, 1968). Scholz montra que les variations de b étaient aussi reliées à la porosité des roches : une roche fortement poreuse aura une plus large proportion de micro-fractures qu'une roche moins poreuse, et donc b sera plus grande pour une roche poreuse. Dans le cas d'un volcan, de petits séismes sont généralement enregistrés et les failles associées sont petites. Les roches volcaniques sont souvent poreuses, et de l'eau sous pression peut circuler dans cette roche poreuse plus facilement. La pression de fluide dans les pores peut diminuer la contrainte normale effective facilitant la rupture de nombreuses micro-fissures et ainsi engendrer de fortes valeurs de b (Scholz, 1968) comme pour la sismicité induite dans les réservoirs (Simpson et al., 1988). Warren \& Latham (1970) ont montré sur des échantillons de roches que des contraintes thermo-élastiques dues à des gradients de température peuvent également générer des valeurs de b supérieures à 1 , comme souvent observé sous les volcans. Warren \& Latham observèrent sur des échantillons de roche que l'activité sismique commençe soudainement juste après une variation de température appliquée sur ces échantillons. Le maximum d'activité correspondant au maximum du gradient thermique. Ils ont également observé la présence d'essaims après la fin apparente de l'activité, corrélée avec de petites fluctuations du gradient thermique.

L'essaim de Quito est localisé sous le vieux volcan Casitagua à 15-20 km au NE du volcan actif du Guagua Pichincha (Fig. 1), où se trouvent de nombreuses failles préexistantes et les roches volcaniques sont souvent poreuses. Le Guagua Pichincha a eu de nombreuses explosions phréatiques et magmatiques peu de temps après l'apparition de l'essaim de Quito. Donc des mouvements de magma sous le volcan ont pu générer des mouvements de fluides (gaz et/ou eau) autour de l'édifice volcanique, changeant le régime local de contraintes et induisant l'essaim de Quito. 
Dans le cas de l'essaim de Quito, l'hypothèse des contraintes thermales pour expliquer une grande valeur de b n'est pas retenue parce que le volcan Casitagua est vieux et le volcan Guagua Pichincha est trop loin pour avoir un effet thermique direct sur cet essaim car la conductivité thermique est en général très faible dans les roches.

Les brisures dans la loi de GR ont déjà été vues pour les grands séismes. Ceci fut expliqué par la dimension finie de la lithosphère sismogénique qui limite l'épaisseur de la zone de rupture. La seule possibilité restante est une extension dans la direction horizontale (Scholz, 1982 ; Pachecæt al., 1992). Comme dans notre cas nous n'avons que des petits séismes $\left(M_{L}<4,2\right)$, nous pouvons supposer que la croissance des failles est auto-similaire (i.e. les failles sont carrées et pas rectangulaires). Ainsi, la cassure dans la loi de GR doit être expliquée d'une autre façon.

La loi de GR montre une double pente très claire (Fig. 2). Nous interprétons ce phénomène comme une bifurcation (au sens de la physique non linéaire) à la suite de laquelle deux comportements sont possibles : une éruption du volcan ou son absence d'activité. Nous spéculons que la tendance d'aller vers une éruption ou non peut être générée par de petites causes, telles que des variations d'amplitude des contraintes dues à une pression de pore du magma ou de l'eau souterraine. Ainsi il est difficile d' utiliser une telle bifurcation comme un paramètre précurseur car le système dynamique peut bifurquer soit vers un scénario catastrophique d'une éruption, ou vers une période de calme du volcan, dépendant de petites variations indétectables ou inprédictibles de la pression.

Une cassure dans les dimensions fractales a déjà été observée durant l'extraction de gaz (Volant et al., 1992). Dans notre étude, nous n'observons qu'une cassure dans la loi de GR et pas dans l'analyse des dimensions multi-fractales. Cette différence peut être due au fait que le nombre de séismes qui intervient dans la cassure de la loi de GR correspond aux événements de magnitude $2,7<\mathrm{M}_{\mathrm{L}}<5,2$, qui sont trop peu nombreux pour affecter les dimensions multi-fractales.

Une décroissance-croissance des dimensions multi-fractales a déjà été montrée pour des séquences de grosses répliques (Legrand et al., 1996). Un comportement similaire est montré ici pour une activité proche d'un volcan, ce qui peut être intéressant pour prédire des pics d'énergie sismique, même si cela ne peut pas être utilisé pour prédire une éruption volcanique.

\section{CONCLUSIONS}

L'essaim de Quito est fortement relié à l'activité du proche $(15-20 \mathrm{~km})$ volcan Guagua Pichincha, et peut être réactivé par des circulations de fluides comme du magma ou de l'eau sous ou autour du volcan.

Une cassure dans la loi de GR est observée et est interprétée comme une bifurcation. Mais elle ne peut cependant pas être utilisée comme un signal fiable d'une activité pré-éruptive du volcan car le système dynamique peut conduire soit vers une séquence catastrophique (une éruption) ou non, dépendant de petites causes comme des variations de pression associées à des mouvements de fluides dans un milieu poreux. 
L'étude de variations temporelles des dimensions multi-fractales semble être plus efficace et précise que des variations temporelles du paramètre b. Nous suggérons que les dimensions multi-fractales décroissent avant les 5 pics principaux d'énergie. Ceci pourraît être utilisé comme un outil pour prédire un pic d'énergie, même si cela ne peut pas être utilisé comme un précurseur fiable des éruptions volcaniques puisque nous interprétons ce phénomène comme un comportement dynamique chaotique et donc imprédictible à long terme.

\section{Remerciements}

Nous remercions le personnel de l'Institut de Géophysique de l'Ecole Polytechnique Nationale de Quito pour le suivi permanent de l'activité sismique et volcanique de l'Équateur et la surveillance 24H/24 durant les crises volcaniques pendant 2 ans. Nous remercions également l'IRD (Institut de Recherche pour le Développement), l'IFEA (Institut Français d'Études Andines) et le MAE (Ministère des Affaires Étrangères) pour le financement d'une partie de cette étude. Nous remercions B. Guillier et Ph. Labazuy pour une revue critique de l'article.

\section{Références citées}

AKI, K., 1965 - Maximum likelihood estimate of $\mathrm{b}$ in the formula $\log \mathrm{N}=\mathrm{a}-\mathrm{bM}$ and its confidence limits. Bulletin of the Earthquake Research Institute; No43: 237-239; University of Tokyo.

AKI, K., 1967 - Scaling low of seismic spectrum. Journal of Geophysical Research; 72: 12171231.

AKI, K., 1981 - A probabilistic synthesis of precursory phenomena. Earthquake Prediction : An international review, Maurice Ewing Ser. (Simpson \& Richards Ed.), Vol. 4: 566-574, Washington: AGU.

BAKER, G., GOLLUB, J., 1990 Chaotic dynamics: an introduction, Cambridge: Cambridge University Press.

BARTON, D., FOULGER, G., HENDERSON, J. \& JULIAN, B., 1999 - Frequency-magnitude statistics and spatial correlation dimensions of earthquakes at Long Valley caldera, California. Geophysical Journal International; 138: 563-570.

BESSE, Ph., 1986 - Relocalisation relative d'événements sismiques appliqué à la région Sud du lac Ghoubbet situé dans la République de Djibouti. Ph'D, 49 pp.

CALAHORRANO, A., YEPES, H., GUILLIER, B., RUIZ, M., SEGOVIA, M., VILLAGÓMEZ, D. \& ANDRADE, D., 1999 - Seismic swarm in Quito (Ecuador) : tectonic or volcanic origin In : Fourth ISAG 119-122; Goettingen: IRD.

CALAHORRANO, A., 2001 - Estudio del origen del enjambre sísmico de la zona norte de la ciudad de Quito, durante 1998-99. Escuela Politécnica Nacional, facultad de geología, minas y petróleos, tesis de ingeniera geóloga, 190p.; Quito.

COUGHLIN, J., Kranz, R., 1991 - New approaches to studying rock burst-associated seismicity in mines. In: Proc. $32^{\text {nd }}$ US sump., Rock Mechanics as a multidisciplinary Science: 491500, Eds. Roegiers, Balkema, Rotterdam.

DONGSHENG, L., ZHAOBI, Z., BINGHONG, W., 1994 - Research into the multifractal of earthquake spatial distribution. Tectonophysics; 233: 91-97.

ECKMANN, JP., RUELLE, D., 1985 - Ergodic theory of chaos and strange attractorsReviews of Modern Physics, 57: 617-656. 
ENEVA, M., 1994 - Monofractal or multifractal : a case study of spatial distribution of mining induced seismic activity. Nonlinear processes in Geophysics, 1: 182-190.

ENEVA, M., BEN-ZION, Y., 1994 - Patterns in synthetic seismicity along faults undergoing brittle and creep deformations, EOS trans., Suppl. 1994 AGU Fall meeting, 75, 462 (abstract).

ENEVA, M., HABERMANN, R. \& HAMBURGER, M., 1994 - Artificial and natural changes in the rates of seismic activity : a case study of the Garm region, Tadjikistan (CIS). Geophysical Journal International; 116: 157-172.

ENEVA, M., 1996 - Effect of limited data sets in evaluating the scaling properties of spatially distributed data : an example from mining-induced seismic activityGeophysical Journal International: 124: 773-786.

FRANCIS, T., 1968a - The detailed seismicity of mid-oceanic ridgesEarth Planet Science Letters: 4: 39-46.

FRANCIS, T., 1968b - Seismicity of mid-oceanic ridges and its relation to properties of the upper mantle and crust, Nature; 220: 899-901.

FRANCIS, T., 1974 - A new interpretation of the 1968 Fernandina caldera collapse and its implications for the Mid-Oceanic ridgesGeophysical Journal of the Royal Astronomical Society; 39: 301-318.

GRASSBERGER, P., 1983 - Generalized dimensions of strange attractors, Physical review Letters; 97 A: 227-230.

GRASSBERGER, P., PROCACCIA, I., 1983 - Measuring the strangeness of strange attractors, Physica; 9 D: $189-208$.

GUTENBERG, B., RICHTER, Ch., 1944 - Frequency of earthquakes in CaliforniaBulletin of the Seismological Society of America; 34: 185-188.

GUTENBERG, B., RICHTER, Ch., 1949 Seismicity of the Earth and associated phenomena. Princeton University Press.

GUTENBERG, B., RICHTER, Ch., 1954 Seismicity of the Earth and associated phenomena. $2^{\text {nd }}$ Edn., Princeton University Press.

HABERMANN, R., 1987 - Man-made changes of seismicity ratesBulletin of the Seismological Society of America; 77: 141-159.

HAVSTAD, J., EHLERS, C., 1989 - Attractor dimension of nonstationary dynamical systemes from small data sets. Physical Review; A, 39: 845-853.

HENTSCHET, H., PROCACCIA, I., 1983 - The infinite number of generalized dimensions of fractals and strange attractors, Physica, $8 \mathrm{D}, 435-444$.

HILL, D., ELLSWORTH, W., JOHNSTON, M., LANGBEIN, J., OPPENHEIMER, D., PITT, A., REASENBERG, P., SOREY, M. \& MCNUTT, S., 1990 - The 1989 earthquake swarm beneath Mammoth Mountain, California : an initial look at the 4 May through 30 September activity. Bulletin of the Seismological Society of America, 80 : 325-339.

HIRATA, T., SATOH, T. \& ITO, K., 1987 - Fractal structure of spatial distribution of microfracturing in rock. Geophysical Journal of the Royal Astronomical Society, 90, 369374.

HIRATA, T., 1989 - A correlation between the b value and the fractal dimension of earthquakes. Journal of Geophysical Research; 94: 7507-7514.

HIRATA, T. \& IMOTO, M., 1991 - Multifractal analysis of spatial distribution of microearthquakes in the Kanto region. Geophysical Journal International; 107: 155-162.

ISHIMOTO, M. \& IIDA, K., 1939 - Observations sur les séismes enregistrés par le microséismographe construit dernièrement (1). Bulletin of the Earthquake Research Institute; 17: 443-478; Tokyo: University of Tokyo.

KAGAN, Y. \& KNOPOFF, L., 1978 - Statistical study of the occurrence of shallow earthquakes. Geophysical Journal of the Royal Astronomical Society; 55: 67-86. 
KAGAN, Y.\& KNOPOFF, L., 1980 - Spatial distribution of earthquakes : the two-point correlation function. Geophysical Journal of the Royal Astronomical Society; 62: 303-320.

KAGAN, Y.\& KNOPOFF, L., 1987 - Statistical short term earthquake predictionScience; 236: 1563-1567.

KING, G., 1983 - The accommodation of large strain in the upper lithosphere of the earth and other solids by self-similar faults systems : The geometrical origin of b-valuePure Appl. Geophys.; 121: 761-815.

KURTHZ, J., HERZEL, H., 1987 - An attractor in solar time seriesPhysica; 25 D: 165-172.

LEE, W., BENNETT R. \& MEAGHER, K., 1972 - A method of estimating magnitudes of local earthquakes from signal duration. Geol. Surv. Open-file Rep 28.

LEGRAND, D., CISTERNAS, A. \& DORBATH, L., 1996 - Multifractal analysis of the 1992 Erzincan aftershock sequence. Geophys. Res. Lett.; 23: 933-936.

LEGRAND, D., CALAHORRANO, A., GUILLIER, B., RIVERA,L., RUIZ, M., VILLAGÓMEZ, D. \& YEPES, H., 2002 - Stress tensor analysis of the 1998-99 tectonic swarm of northern Quito related to the volcanic swarm of Guagua Pichincha volcano, Ecuador. Tectonophysics; 344, 15-36.

MAIN, I., 2000 - Apparent breaks in scaling in the earthquake cumulative frequency-magnitude distribution : fact or artifact Bulletin of the Seismological Society of America; 90: 8697.

MANDELBROT,B., 1975 Les objetsfractals :forme, hasard et dimensionFlammarion, Paris.

MATSUMURA, S., OHKUBO, T. \& IMOTO, M., 1991 - Seismic swarm activity in and around the Izu Peninsula preceding the volcanic eruption of July 13, 1989. Journal of Physics of the Earth; 39: 93-106.

MCNUTT, S., 1986 - Observations and analysis of B-type earthquakes, explosions and volcanic tremor at Pavlof volcano, Alaska. Bulletin of the Seismological Society of America; 76: 153-175.

MCNUTT, S., 2000 Seismic monitoring, in Encyclopedia of volcanoes, Haraldur Sigurdsson (Ed.), Academic Press

MOGI, K., 1963 - Some discussions on aftershocks, foreshocks, and earthquake swarms- The fracture of a semi-infinite body caused by an inner stress origin and its relation to the earthquake phenomena, 3, Bulletin of the Earthquake Research Institute; 41: 615-658; Tokyo: University of Tokyo.

MOGI, K., 1969 - Some features of recent seismic activity in and near Japan (2) Activity before and after great earthquakes. Bulletin of the Earthquake Research Institute; 47: 395-417; University of Tokyo, .

OGATA, Y., 1988 - Statistical models for earthquakes occurrences and residual analysis for point processes. Journal of the American Statistical Association; 83: 401.

PACHECO, J., SCHOLZ, C. \& SYKES, L., 1992 - Changes in frequency-size relationship from small to large earthquakes, Nature; 355: 71-73.

POUPINET, G., ELLSWORTH, W. \& FRÉCHET, J., 1984 - Monitoring velocity variation in the crust using earthquake doublets : an application to the Calaveras fault, CaliforniaJ. Geophys. Res.; 89, 5719-5731.

POWER J., WYSS, M. \& LATCHMAN J., 1998 - Spatial variations in the frequency-magnitude distribution of earthquakes at Soufriere Hills volcano, Montserrat, West Indies. Geophys. Res. Let.; 25: 3653-3656.

RADULIAN, M. \& TRIFU, C., 1991 - Would it have been possible to predict the 30 August 1986 Vrancea earthquake Bulletin of the Seismological Society of America; 81: 2498-2503.

RIVERA, L., 1989 - Inversion du tenseur des contraintes et des mécanismes au foyer à partir des données de polarités pour une population de séismes. Application à l'étude du foyer de sismicité intermédiaire de Bucaramanga (Colombie). Thèse de doctorat, Univ. Strasbourg, France, 266 pp. 
SCHOLZ, C., 1968 - The frequency-magnitude relation of microfracturing in rock and its relation to earthquakes. Bulletin of the Seismological Society of America; 58: 399-415.

SCHOLZ, C., 1982 - Scaling laws for large earthquakes : consequences for physical models, Bulletin of the Seismological Society of America; 72: 1-14.

SCHOLZ, C., 1994 - A reappraisal of large earthquake scaling Bulletin of the Seismological Society of America; 84: 215-218.

SEGOVIA, M., 2001 - El sismo de Bahía del 4 de Agosto de 1998 : Caracterización del mecanismo de ruptura y analisis de la sismicidad en la zona Costera. Dipl. Ing., Escuela Politécnica Nacional, Quito, 136 pp.

SIMPSON, D., LEITH, W. \& SCHOLZ, C., 1988 - Two types of reservoir-induced seismicity. Bulletin of the Seismological Society of America; 78: 2025-2040.

SMITH, W., 1981 - The b-value as an earthquake precursorNature; 289: 136-139.

SORNETTE, A., SORNETTE, D., 1999 - Renormalization of earthquake aftershocksGeophys. Res. Let.; 26: 1981-1984.

SPENCE, W., 1980 - Relative epicenter determination using P-wave arrival time differences. Bulletin of the Seismological Society of America; 70: 171-183.

SUYEHIRO, S., ASADA, T. \& OHTAKE, M., 1964 - Foreshocks and aftershocks accompanying a perceptible earthquake in central Japan. Paper Meteorol. Geophys.; 15: 17-88.

SYKES, 1970 - Earthquake swarm and sea-floor spreadingJ. Geophys. Res.; 75: 6598-6611.

TURCOTTE, D., 1992 Fractals and chaos in geology and geophysics. Cambridge U. Press, New York.

VILLAGÓMEZ, D., RUIZ, M., YEPES, H., HALL, M., GUILLIER, B., ALVARADO, A., SEGOVIA, M. \& CALAHORRANO, A., 1999 - Seismic activity at Guagua Pichincha volcano, Ecuador. Fourth ISAG, Goettingen (Germany), 793-796.

VILLAGÓMEZ, D., 2000 - Sismicidad del volcán Guagua Pichincha, 1998-1999. Escuela Politécnica Nacional, facultad de geología, minas y petróleos, tesis de ingeniero geólogo, Quito, Ecuador, 114 pp.

VOLANT, Ph., GRASSO, JR, CHATELAIN, JL \& FROGNEUX, M., 1992 - b-value, aseismic deformation and brittle failure within an isolated geological object : evidences from a dome structure loaded by fluid extraction, Geophys. Res. Lett.; 19: 1149-1152.

WARREN, N., LATHAM, G., 1970 - An experimental study of thermally induced microfracturing and its relation to volcanic seismicity. J. Geophys. Res.; 75: 4455-4464.

WESNOUSKY, S., 1999 - Crustal deformation processes and the stability of the GutenbergRichter relationship. Bulletin of the Seismological Society of America; 89: 1131-1137.

WIEMER, S., WYSS, M., 1997 - Mapping the frequency-magnitude distribution in asperities an improved technique to calculate recurrence times Journal of Geophysical Research; 102: $15115-15128$.

WIEMER, S., MCNUTT, S., 1997 - Variations in the frequency-magnitude distribution with depth in two volcanic areas : Mount St. Helens, Washington, and Mt. Spurr, Alaska. Geophysical Research Letters; 24: 189-192.

WIEMER ,S., MCNUTT, S. \& WYSS, M., 1998 - Temporal and three-dimensional spatial analyses of the frequency-magnitude distribution near Long Valley Caldera, California. Geophys. J. Int., 134, 409-421.

WYSS M., 1973 - Towards a physical understanding of the earthquake frequency distribution. Geophys. J. R. Astr. Soc., 31, 341-359.

WYSS M., HABERMANN R., 1988 - Precursory seismic quiescence. Pageoph, 126, 319-332.

WYSS, M., SHIMAZAKI, K., WIEMER, S., 1997 - Mapping active magma chambers by b-value beneath Off-Izu volcano, Japan. Journal of Geophysical Research, 102, 20413-20433. 